\title{
The Role of Current Audiological Tests in the Early Diagnosis of Hearing Impairment in Infant
}

\author{
Seikholet Kuki $\cdot$ Shelly Chadha $\cdot$ Shruti Dhingra $\cdot$ \\ Achal Gulati
}

Received: 18 March 2012/ Accepted: 26 March 2012/Published online: 25 August 2012

(C) Association of Otolaryngologists of India 2012

\begin{abstract}
This study aims to compare the various audiological tests that are currently available as screening tools for infant hearing assessment. In developing countries, which have limited resources, it is imperative to design a screening programme that is easy to perform, gives accurate results, has good patient acceptability and is cost effective. Fifty infants, 3-12 months of age, were subjected to BOT for hearing, transient-evoked oto-acoustic emissions (TEOAEs), automated auditory brainstem response (AABR) test, auditory brainstem evoked response (ABR) testing and auditory steady state response (ASSR). The sensitivity and specificity of the various tests was calculated, with ABR test as the gold standard. We also compared them for average time taken, cost factors, ease of performance, number of repeat tests required and patient acceptability. All the screening tests, TEOAE, AABR and behavioral observation test had reasonably high sensitivity. Specificity was highest for behavioral observation testing $(67.7 \%)$ and AABR (64.5\%), but low for OAE test $(48.3 \%)$. The cost and time taken for each test was least for the behavioral observation test, which also did not require any repeat testing. TEOAE and AABR incur a recurring cost of approximately one dollar per test. However, in 12 and $28 \%$ of the babies had to be called back for repeat testing, respectively. ASSR, as a diagnostic test, has a $100 \%$ sensitivity and high specificity but scores down on the cost involved, time taken and the number of repeat tests
\end{abstract}

S. Kuki $\cdot$ S. Chadha $\cdot$ S. Dhingra $(\bowtie) \cdot$ A. Gulati Department of Otolaryngology, Head and Neck Surgery, Maulana Azad Medical College and Associated Lok Nayak Hospital, New Delhi, India

e-mail: shrudoc@hotmail.com required. Overall parent acceptability was the highest for behavioral observation test, as it was non-invasive and easy to perform. Behavioral observation combined with TEO$\mathrm{AE} / \mathrm{AABR}$, can be fairly accurate as screening tests for hearing loss in infants. Considering its ease of performance and low cost, behavioral observation could be used as a screening test for detection of hearing loss in infants, in those countries/places where universal neonatal hearing screening is not yet available.

Keywords Audiological tests · Hearing impairment . Infant screening

\section{Introduction}

The ears and the eyes are the portals of learning and communication. Appreciation of thoughts and words is difficult unless they are heard and understood. Universal newborn hearing screening programs have shown that permanent hearing loss is one of the most common abnormalities present at birth. In 1999, the American Academy of Paediatrics Task Force on Newborn and Infant Hearing [1] stated, "Significant bilateral hearing loss has been shown to be present in approximately 1-3 per 1,000 newborns in the well-baby nursery population, and in approximately $2-4$ per 1,000 infants in the intensive care unit population." Almost $50 \%$ of deafness and hearing impairment are preventable especially in the developing countries. Hence there is an urgent need to develop national programmes for hearing screening to accurately identify infants with significant hearing impairment in the most rapid and cost-effective way that will minimize referral rates and maximize sensitivity and specificity. With these goals in mind, the NIH Consensus Statement recommends that all screening programs 
should have a failure rate of no more than 5-7\%, and the number of referrals be kept to a minimum [2].

The purpose of this study was to assess the sensitivity and specificity of various techniques used in hearing screening of infants-behavioural observation testing (BOT), transientevoked oto-acoustic emission (TEOAE), auditory steady state response (ASSR) and brain stem evoked response audiometry (BERA), automated auditory brainstem responses (AABR). We also aimed to study these tests with reference to time taken, cost factors and patient acceptability.

\section{Research Design and Methods}

\section{Subjects}

The study was conducted in the department of Otorhinolaryngology and Head and Neck Surgery in association with the department of Pediatrics in Lok Nayak Hospital, New Delhi. Infants between 3 to 12 months of age were taken from the ENT Out Patient Department and the High risk baby clinic. A total of 50 babies (35 male and 15 female) were enrolled in the study who had history suggestive of one or more of the risk factors mentioned below.

1. Family history of childhood hearing impairment

2. Congenital perinatal infection (e.g., Cytomegalovirus, Rubella, herpes, toxoplasmosis, syphilis)

3. Anatomic malformations involving the head or neck

4. Birth weight $<1,500 \mathrm{~g}$.

5. Hyperbilirubinemia requiring exchange transfusion

6. History of bacterial meningitis especially Haemophilus influenza

7. History of birth asphyxia with APGAR scores of $0-4$ at $1 \mathrm{~min}$ or $0-6$ at $5 \mathrm{~min}$.

8. Hospitalization in neonatal ICU greater than $48 \mathrm{~h}$

Infants excluded were those with external auditory canal abnormalities, presence of impacted wax, debris or foreign bodies that could not be removed, ear discharge, abnormalities of tympanic membrane, any child who did not complete the study and those not willing to give consent. After obtaining an informed consent from the parent, each infant was assessed for demographic profile, prenatal, perinatal and postnatal history, milestones in relation to hearing, family history and congenital abnormalities.

Each infant underwent testing with BOT, TEOAE, AABR, ASSR and BERA in random order.

\section{BOT}

A total of 50 infants aged 3-12 months were subjected to BOT. The methodology used for the test was based on the methodology described within the WHO Trainer's manual of the Primary ear \& hearing Care training resource [3]. It was performed in an OPD setup with ambient noise levels $<30 \mathrm{~dB}$. Two testers, one to present the sound stimuli and other as distractor in front of the child. The test was performed with the child cradled in the parent's lap, facing forward and erect, lightly supported around the waist. The child's attention was lightly engaged in front by a distractor. The sound stimulus was presented for $<2 \mathrm{~s}$, in a horizontal plane, $15 \mathrm{~cm}$ from the child's ear, out of peripheral vision half a second after the item was uncovered. The distractor observed evidence of a response.

The stimulus was given in the form of a noise maker or rattle (less frequency specific). Initially a lower stimulus of approximately $40 \mathrm{~dB}$ was presented. If there was no response this was increased and a louder stimulus approximately $70-80 \mathrm{~dB}$ was presented. If this elicited no response a louder stimulus approximately $100 \mathrm{~dB}$ was presented with the change of two metallic objects. Both sides of the ear were tested separately. It is a quick and rough screening method before one embarks on a more eletrophysiological method of objective assessment.

The sound stimulus was presented for approximately $2 \mathrm{~s}$, in a horizontal plane, $15 \mathrm{~cm}$ from the child's ear, out of peripheral vision. The Noisemaker was held at an angle of $45^{\circ}$ to the horizontal plane, both sides were tested separately.

The behavioural response was taken as "Pass" if eye widening, eye blink (auropalpebral reflex), arousal from sleep, startle or shudder of the body or definite movement of the arms, legs or body or Lateral inclination of the head towards the sound or a listening attitude or stilling or definite eye movement/listening/turning of the head towards the sound was present. If all of these are absent the result is considered as "Refer".

The observer's bias (dependency) was reduced by way of a single individual doing all the tests. The observer was blinded to the results of the other tests.

\section{TEOAE}

With the first visit to the hospital the baby with known risk factors were screened with TEOAEs in both ears separately, in either asleep or awake state in a quiet environment. It employed click stimuli at $\sim 80-85-\mathrm{dB}$ SPL with the stimulation rate 50-60 stimuli per second. Acoustic events were recorded in a $16 \mathrm{~ms}$ time window starting $5 \mathrm{~ms}$ after stimulus onset. A computerized averaging system obtained a clean recording. The results were shown on the screen as either 'Pass' or 'Refer'. In case of a 'refer' to the test ear a repeat testing was done up to a third time to be sure it was not due to faulty technique, inappropriate size probe or insertion technique. 
Irrespective of the results of the BOT and the TEOAE, the individual patient was tested with the AABR. For all the electrophysiological tests [AABR, auditory brainstem evoked response (ABR) and ASSR] every child was sedated with suspension Pedichloryl (Triclorofos, $0.3 \mathrm{ml} / \mathrm{kg}$ body weight) and the child was taken to a sound attenuated room. Otoscopy was done to confirm that the ear canal was clean. The baby was made to lie supine with head stabilized on a soft thin pillow. The forehead, preauricular and the post auricular regions were cleaned with spirit soaked cotton swab and then let dried.

\section{AABR Test}

The non-portable MB2 BERA phone software screener was used. It uses $35 \mathrm{~dB}$ near hearing level click stimuli, presented mono-aurally at rate of 37 pulses/s through head phone with scalp electrodes.

AABR measurements were obtained by placing metallic silver surface electrodes on the forehead (recording electrode), on the ipsilateral mastoid process (reference electrode), and on the contralateral mastoid process (ground electrode). The click stimulus (set at $35 \mathrm{~dB}$ hearing level [HL]) was delivered to the infant's ear via head phones designed to attenuate background noise. A pass or fail response was obtained.

\section{Auditory Brainstem Evoked Response Audiometry} (BERA)

Settings for ABR acquisition: using SmartEP of the Intelligent Hearing System SNSEP010 1-800-IHSYSTEMS. A click stimulus at the rate of 19.3 or $21.1 /$ s was given for $0.1 \mathrm{~ms}$. Insert Earphone with an amplification of $\times 100$ were used in the intensity range of $90 \mathrm{~dB} H \mathrm{HL}$ down to $0 \mathrm{~dB}$ HL. The Analysis Time Window $=12.8 \mathrm{~ms}$. The waves were recorded and analysed. Analysis was done by the machine but was also checked manually.

\section{ASSR}

Using Smart-EP of the Intelligent Hearing system (IHS) SNSEP010 1-800-IHSYSTEMS like in BERA the default programme of ASSR, smartEP was set up and the profile of each patient entered.

The test was done within the frequency range of 500, 1 , 2 and $4 \mathrm{kHz}$ spectra. The intensity of the stimulus was increased or decreased with $10 \mathrm{~dB}$ up/down, with 420 sweeps. Both the ears were tested simultaneously with insert earphones as a stimulator to deliver acoustic stimuli. Noise rejection level was set at $31 \%$.

\section{Results}

All the children included in the study were those who had suffered some risk factor during antenatal, perinatal or postnatal period. The most common risk factor was low birth weight $<1500 \mathrm{~g}$ in 22 patients followed by hyperbilirubinemia in 16 patients. Most of the children in the study had only a single risk factor. However, ten babies were exposed to two risk factors. 41 of the babies had a history of delayed language milestones. 32 out of these 41 infants also had a concurrent delay in developmental milestones. Nine of those with delayed hearing milestone had normal development.

Screening tests: Table 1

\section{BOT}

Of the 100 ears tested, 75 showed no/doubtful response on testing while 25 ears showed positive response on behavioral testing. Of the 50 infants tested 39 had failed the test. Three infants failed in one ear and 36 of them failed in both ears.

\section{TEOAE}

Of the 100 ears screened 82 ears had failed the procedure and 18 ears passed the test by OAE. Of the 50 infants screened by this method 39 failed the test in both the ears, 3 failed in the left ear and 1 failed in the right ear.

\section{$A A B R$}

AABR test was done on 50 infants in both ears separately. Out of the 100 ears screened 76 ears failed the test and 24 passed the screening test by this method. There was no disparity between the two ears and the 76 failures belonged to 38 babies.

Diagnostic tests: Table 2

\section{$A B R$}

Out of the 100 ears tested 33 infants showed presence of normal waveforms at $35 \mathrm{~dB}$. Whereas in the rest of the 67

Table 1 Result of screening of 100 ears

\begin{tabular}{llll}
\hline $\begin{array}{l}\text { Screening } \\
\text { test }\end{array}$ & $\begin{array}{l}\text { Number of ears } \\
\text { screened }\end{array}$ & $\begin{array}{l}\text { Number of ears } \\
\text { referred }\end{array}$ & $\begin{array}{l}\text { Number of ears } \\
\text { passed }\end{array}$ \\
\hline BOT & 100 & 75 & 25 \\
OAE & 100 & 82 & 18 \\
AABR & 100 & 76 & 24 \\
\hline
\end{tabular}


Table 2 Result of ABR testing

\begin{tabular}{lll}
\hline $\begin{array}{l}\text { No. of ears } \\
\text { tested }\end{array}$ & $\begin{array}{l}\text { No. of ears showing } \\
\text { normal wave V at } 35 \mathrm{~dB}\end{array}$ & $\begin{array}{l}\text { Delayed or absent } \\
\text { wave V }\end{array}$ \\
\hline 100 & 67 & 33 \\
\hline
\end{tabular}

tested ears showed delayed or absent Wave V. These 67 ears belonged to 34 children. 33 children failed the test in both the ears, while one infant with a family history of deafness had normal wave patterns in left ear, but revealed no response in the right ear.

\section{ASSR}

The patients were divided into four groups. Hearing thresholds were studied in the frequency range 0.5, 1, 2 and $4 \mathrm{kHz}$. Patients with their hearing threshold better than $20 \mathrm{~dB}$ in the ASSR audiogram frequencies of $0.5,1,2$ and $4 \mathrm{kHz}$, were placed in the normal hearing group, between $20-40 \mathrm{~dB}$ as mild, $40-60$ as moderate, between $60-80 \mathrm{~dB}$ as severe and above $80 \mathrm{~dB}$ as profound. In this study, out of 100 ears tested 30 infants showed their hearing sensitivity within normal limits, 3 showed mild and 18 children had moderate hearing loss, 12 had severe hearing loss and the remaining 37 had profound hearing loss (Table 3 ).

Based on the results, sensitivity and specificity of each test was calculated. Among the screening tests available the TEOAE is most sensitive while there is small or no difference between AABR and BOT methods in their sensitivity. However, TEOAE has the lowest specificity among the three tests. ASSR has the highest sensitivity and specificity and is comparable to ABR (Table 4).

\section{Other Parameters}

While performing these tests the time taken for each test was also recorded. The time was measured from the moment the baby was brought in for the test till the time that the test was successfully completed. The time for quietening down the baby, application of earphones and electrodes was included.

Time taken for each test is given in Table 5 .

The minimum average time was required for the performance of the BOT and TEOAE tests, while ASSR was the test requiring maximum time. $48 \%$ of children undergoing $\mathrm{ABR}$ test were required to make a repeat visit

Table 3 Result of ASSR testing

\begin{tabular}{llllll}
\hline $\begin{array}{l}\text { No. of ears } \\
\text { tested }\end{array}$ & $\begin{array}{l}\text { Normal } \\
\text { hearing }\end{array}$ & $\begin{array}{l}\text { Mild } \\
\text { H. loss }\end{array}$ & $\begin{array}{l}\text { Moderate } \\
\text { H. loss }\end{array}$ & $\begin{array}{l}\text { Severe } \\
\text { H. loss }\end{array}$ & $\begin{array}{l}\text { Profound } \\
\text { H. loss }\end{array}$ \\
\hline 100 & 30 & 3 & 18 & 12 & 37 \\
\hline
\end{tabular}

in order for the test to be completed. $52 \%$ of the children who underwent ASSR had to come more than once for the test to be completed. 28 and $12 \%$ of those undergoing AABR and TEOAE, respectively had to revisit of the test to be completed satisfactorily.

\section{Cost Factors}

The cost factor of the test was calculated. This was based on the initial investment required for the purchase of the machine and infrastructure. Recurring costs included the cost of the disposables used in the test.

The costs calculated were given in Table 6 .

\section{$B O A$}

The behavioral observation test was performed using a noisemaker and toy/torch for distraction. The total initial investment for this test was seventy rupees there was no recurrent cost involved.

\section{$O A E$}

The machine for OAE test was a hand held machine which was procured at an initial cost of Rs. 2,50,000. The disposables used included the ear phones. The same set of ear phones was used repeatedly and not disposed of after each test. The average cost per patient was Rs. 40 .

\section{$A A B R$}

The combined machine for AABR, ABR and ASSR test was procured by the hospital at a cost of Rs. 14, 00,000. However, in AABR test, ultragel, cotton and spirit was used. The average cost per patient was worked out to be Rs. 45.

\section{$A B R$ and ASSR Test}

The recurring expense of ABR and ASSR tests includes the electrodes used along-with the electrode jelly, insert earphones, cotton and spirit. The fact that a lot of children had to undergo repeat testing also added to the cost. The average cost for this test was computed to be Rs. 200, which is similar to the cost of ASSR. It may be noted that the earphones were mostly reused (due to shortage), a fact that brought down the cost of the test.

\section{Patient's Acceptability: Table 7}

During each test patient's acceptability was assessed with a simple questionnaire. This was based on whether the test 
Table 4 Comparison among the test with regard to sensitivity and specificity
Table 5 Time taken for each test

\begin{tabular}{lllll}
\hline $\begin{array}{l}\text { Test } \\
\text { method }\end{array}$ & $\begin{array}{l}\text { Sensitivity } \\
(\%)\end{array}$ & $\begin{array}{l}\text { Specificity } \\
(\%)\end{array}$ & $\begin{array}{l}\text { Positive predictive value } \\
(\%)\end{array}$ & $\begin{array}{l}\text { Negative predictive value } \\
(\%)\end{array}$ \\
\hline BOT & 94.2 & 67.7 & 86.66 & 84 \\
OAE & 97.1 & 48.3 & 80.72 & 88.23 \\
AABR & 94.20 & 64.51 & 85.52 & 83.33 \\
ASSR & 100 & 96.77 & 98.57 & 100 \\
\hline
\end{tabular}

\begin{tabular}{lllll}
\hline $\begin{array}{l}\text { Test method } \\
\text { used }\end{array}$ & $\begin{array}{l}\text { Minimum time taken } \\
(\mathrm{min})\end{array}$ & $\begin{array}{l}\text { Maximum time taken } \\
(\mathrm{min})\end{array}$ & $\begin{array}{l}\text { Average time } \\
(\mathrm{min})\end{array}$ & $\begin{array}{l}\text { No. of infants requiring } \\
\text { repeat testing }\end{array}$ \\
\hline BOT & 1 & 4 & 3.32 & 0 \\
OAE & 2 & 9 & 4.25 & 6 \\
AABR & 2 & 10 & 5.46 & 14 \\
ABR & 20 & 48 & 32.84 & 24 \\
ASSR & 20 & 60 & 39.80 & 26 \\
\hline
\end{tabular}

Table 6 Cost factors

\begin{tabular}{llll}
\hline Test & Initial investment (USD/INR) & $\begin{array}{l}\text { Average recurring expenditure } \\
(\text { USD/INR) }\end{array}$ & Disposables used \\
\hline BOT & $1.5 / 70$ (noise maker and toy/torch) & 0 & \\
OAE & $6,000 / 2,50,000$ (hand held machine) & $0.8 / 40$ & Ear plugs \\
AABR & $34,000 / 14,00,000$ (combined machine) & $1 / 45$ & Ultra gel, cotton, spirit \\
ABR & & $4.5 / 200$ & Ear plugs, electrodes, spirit, cotton, electrode gel \\
ASSR & $4.5 / 200$ & Ear plugs, electrodes, spirit, cotton, Ten20 electrode gel \\
\hline
\end{tabular}

Table 7 Comparison among the test with regard to sensitivity, specificity, average time taken and cost involved

\begin{tabular}{lllllc}
\hline Test method & Sensitivity $(\%)$ & Specificity $(\%)$ & Time taken (min) & Recurring cost incurred USD/INR & No. of repeat test reqd. \\
\hline BOT & 94.2 & 67.7 & 3.22 & 0 & 0 \\
OAE & 97.1 & 48.3 & 4.25 & $0.8 / 40$ & 6 \\
AABR & 94.20 & 64.51 & 5.46 & $1 / 45$ & 14 \\
ABR & Reference test & Reference test & 32.84 & $4.5 / 200$ & 24 \\
ASSR & 100 & 96.77 & 39.80 & $4.5 / 200$ & 26 \\
\hline
\end{tabular}

could be completed at one go or had to be repeated. It also considered whether the parent was comfortable with the test and would be ready to have it repeated. Subjective test of BOT was well accepted. This may be due to the fact that the child was not subjected to any form of bodily manipulation. All the other tests necessarily required bodily manipulation such as inserting the ear phone into the canal, application of surface electrodes and a higher intensity of sound stimuli made them feel uncomfortable. Also, longer the time taken for the test, more were the chances of failure to complete the test and hence reduced acceptability.

The overall best performance in this study was the BOT. It has a high sensitivity; moderate specificity with practically no cost involved and takes a very short time to complete. Moreover, there was no baby who required a second visit for repeat testing. ASSR performed extremely well in terms of sensitivity and specificity. However in terms of the cost involved, time taken and the number of 
repeat tests required, it is not suitable for routine use on infants with hearing impairment.

\section{Discussion}

The present study was carried out on 50 infants with the aim of determining the sensitivity and specificity of each test and also considering the cost factor, time taken and patients' acceptability.

\section{Behavioral Observation Test}

In our series, the sensitivity of this test was calculated to be over $94 \%$ and the specificity was $67 \%$. The test appears to have a good positive and negative predictive value. The advantages of this method are that the whole auditory pathway is tested. However it is dependent on subjective interpretations. Criteria for presence or absence of reaction are somewhat subjective [4]. The high sensitivity reported for this test in the current study could be due to the fact that all the tests were conducted by the same person who was well trained.

\section{TEOAE}

It is a very convenient screening method with quick results in about 4-5 min time. The major limitation of TEOAE lies in the fact that children who have non-organic hearing loss or hearing loss primarily due to involvement of the auditory nerve may however have completely normal TEOAEs.

In the present study, a total of 100 ears were tested. 83 ears failed the TEOAE test and only 17 ears passed the test. Out of 83 that failed OAE, 67 ears showed significant hearing loss on ABR test. Out of 69 ears that were determined as having hearing loss (by ABR), two ears had passed the TEOAE test. Moreover 16 ears that failed TEOAE test actually had normal hearing pattern on ABR test. In a study performed by Bonfils et al. [5] 98 out the 100 ears passed the TEOAE testing.

\section{AABR}

The Sensitivity was as high as $94.20 \%$, the specificity was $64.51 \%$. The test had a positive predictive value of $85.52 \%$ and a negative predictive value of $83.33 \%$. On the whole, this is a sensitive test that will detect hearing impairment and can be used in a screening programme. Used together, both TEOAE and AABR can increase the sensitivity and specificity which is an ideal solution for screening infants for hearing assessment. In the present study, when we combined the results of both these tests, the sensitivity was over $97 \%$. However, when we decided to test children who failed either of the two tests, the specificity was reduced to about $42 \%$.

\section{ASSR}

Sensitivity of this test was $100 \%$ with specificity of $96.77 \%$, and positive predictive value: $98.57 \%$ and negative predictive value: $100 \%$. Luts et al. [6] made a comparative study between ABR threshold and ASSR thresholds at $2 \mathrm{kHz}$, since the spectrum of the click stimulus contains most energy at frequencies around $2 \mathrm{kHz}$ and found that the correlation of ABR thresholds and ASSR thresholds at $2 \mathrm{kHz}$ to be 0.77 . Only $58 \%$ of the variance of the ABR threshold was explained by its linear relationship with the ASSR threshold.

In the present study comparing ASSR with the ABR which is the gold standard test method, the ASSR could correctly define the hearing status of the baby with deafness This would be an ideal objective test method alternative to $\mathrm{ABR}$, which needs an audiologist to interpret the result, which is to some extent, subjective in nature.

\section{$\mathrm{ABR}$}

$\mathrm{ABR}$ is normally recognizable down to intensities that are $30 \mathrm{~dB}$ above normal hearing thresholds. In general, ABR exhibits a sensitivity of over $90 \%$ and a specificity of approximately 70-90\% [7].

In the present study, we have referred to ABR test as the Gold standard test and the performance of all other tests have been compared to ABR results. As can be observed, amongst the screening tests, TEOAE has the highest sensitivity, but the lowest specificity, while behavioral observation test and AABR have comparable sensitivity and specificity ranges.

Based on the above results, it would seem appropriate to suggest that while the electrophysiological tests of TEOAE and AABR are good screening tests, there are certain problems associated with these tests. TEOAE has a comparatively low specificity (48.3\%), as compared to other screening tests. This fact would mean that in a screening programme using TEOAE alone, there would be a high number of referrals. The specificity can possibly be improved by repeating the TEOAE test at least once in those who fail it the first time [8].

However, that procedure too would increase the number of visits required to be made by the patient and put a greater load on the hospital audiological services. While AABR has a higher specificity as compared to TEOAE, $28 \%$ of the children in our study could not be tested on their first visit and had to be called back for the completion of the test. This matter also added to the cost of the test. 
Amongst the screening tests evaluated, behavioral observation test has high sensitivity and reasonable specificity. However, what may be a potential limiting factor to the widespread application of this test as a screening tool is the competence level of the Observer who carries out this test. A similar or even higher competence level is also required of the person who interprets the ABR test results, as identification of Wave $\mathrm{V}$ can be a subjective issue. Thus, at times, ASSR emerges as an excellent diagnostic tool with $100 \%$ sensitivity and a high specificity to match. AABR takes only about 2 min longer than BOT on an average. Since it is automated, an audiologist could train multiple health care personnel to set up and perform the test, relieving the need to have a single specialized audiologist for each patient which is required for BOT.

\section{Conclusion}

The overall patient acceptability was excellent for BOT and good for TEOAE. This may be due to the fact that they can be easily performed in the out-patient setting, are less time consuming, without the need to sedate the child. In developing countries, where the more objective test machines are not available at the primary level, BOT remains an attractive option with almost zero recurring cost and high sensitivity and specificity. ASSR and ABR shall continue to serve as diagnostic tools to confirm or refute the results of the screening tests. It is however not advisable to use them as screening procedures, in view of the cost involved, patient preparation, time constraints and manpower. While electrophysiological testing is undoubtedly the best option for infant hearing screening, BOT can provide a viable alternative as a first level screening tool. Combined with TEOAE, it can as a first stage protocol in a developing country with a typical rural set-up.

\section{References}

1. American Academy of Pediatrics (1999) Task force on newborn and infant hearing. Newborn and infant hearing loss: detection and intervention. Pediatrics 103(2):527-530

2. Early identification of hearing impairment in infants and young children. NIH Consens Statement, 1-3 Mar 1993, vol 11(1), pp 1-24

3. Primary Ear \& Hearing Care Training Resource (2006) Intermediate level trainer's manual. World Health Organization. http://www. who.int/pbd/deafness/activities/hearing_care/intermediate.pdf

4. Robertson C, Aldridge S, Jarman F, Saunders K, Poulakis Z, Oberklaih F (1995) Late diagnosis of congenital sensorineural hearing impairment-why are detection methods failing? Arch Dis Child 72:11-15

5. Bonfils P, Dumont A, Marie P, Francoise M, Narcy P (1990) Evoked otoacoustic emissions in newborn screening. Laryngoscope 100:186-189

6. Luts H, Desloovere C, Kumar A, Vandermeersch E, Wouters J (2004) Objective assessment of frequency-specific hearing thresholds in babies. Int J Pediatr Otorhinolaryngol 68(7):915-926

7. Schmidt RJ, Sataloff RT, Newman J, Spiegel JR, Myers DL (2001) The sensitivity of auditory brainstem response testing for the diagnosis of acoustic neuromas. Arch Otolaryngol Head Neck Surg 127(1):19-22

8. Johnson JL, White KR, Widen JE, Gravel JS, James M, Kennalley T, Maxon AB, Spivak L, Sullivan-Mahoney M, Vohr BR, Weirather Y, Holstrum J (2005) A multicenter evaluation of how many infants with permanent hearing loss pass a two-stage otoacoustic emissions/ automated auditory brainstem response newborn hearing screening protocol. Pediatrics 116:663-672 